\title{
Is Ki67 prognostic for aggressive prostate cancer? A multicenter real-world study
}

\author{
Joseph J Fantony1, Lauren E Howard ${ }^{2,3}$, Ilona Csizmadi ${ }^{4}$, Andrew J Armstrong ${ }^{5}$, Amy L \\ Lark $^{6,7}$, Colette Galet ${ }^{8,9}$, William J Aronson ${ }^{8,10}$ \& Stephen J Freedland ${ }^{2,4}$ \\ ${ }^{1}$ Department of Surgery, Duke University Medical Center, Durham, NC 27710, USA \\ 2Urology Section, Durham Veterans Affairs Medical Center, Durham, NC 27705, USA \\ ${ }^{3}$ Department of Biostatistics and Bioinformatics, Duke School of Medicine, Durham, NC 27705, USA \\ ${ }^{4}$ Department of Surgery, Cedars-Sinai Medical Center, Los Angeles, CA 90048, USA \\ ${ }^{5}$ Department of Medicine, Duke University School of Medicine, Durham, NC 27710, USA \\ ${ }^{6}$ Department of Pathology, Duke University Hospital, Durham, NC 27710, USA \\ ${ }^{7}$ Department of Pathology, Durham Veterans Affairs Medical Center, Durham, NC 27710, USA \\ ${ }^{8}$ Department of Urology, David Geffen School of Medicine, University of California at Los Angeles, Los Angeles, CA 90095, USA \\ ${ }^{9}$ Division of Acute Care Surgery, Department of Surgery, Carver College of Medicine, University of lowa, IA 52242, USA \\ ${ }^{10}$ Urology Section, Department of Surgery, Greater Los Angeles Veterans Affairs Healthcare System, CA 90073, USA. \\ *Author for correspondence: Tel.: +1 310423 3497; Fax: +1 310423 4711; stephen.freedland@cshs.org
}

\begin{abstract}
Aim: To test if Ki67 expression is prognostic for biochemical recurrence (BCR) after radical prostatectomy (RP). Methods: Ki67 immunohistochemistry was performed on tissue microarrays constructed from specimens obtained from 464 men undergoing RP at the Durham and West LA Veterans Affairs Hospitals. Hazard ratios (HR) for Ki67 expression and time to BCR were estimated using Cox regression. Results: Ki67 was associated with more recent surgery year $(p<0.001)$, positive margins $(p=0.001)$ and extracapsular extension ( $p<0.001$ ). In center-stratified analyses, the adjusted HR for Ki67 expression and BCR approached statistical significance for west LA (HR: 1.54; $p=0.06)$, but not Durham (HR: 1.10; $p=0.74)$. Conclusion: This multi-institutional 'real-world' study provides limited evidence for the prognostic role of Ki67 in predicting outcome after RP.
\end{abstract}

First draft submitted: 25 September 2017; Accepted for publication: 14 February 2018; Published online: 15 June 2018

Keywords: biochemical recurrence • castration-resistant prostate cancer • immunohistochemistry • Ki67 - metastases • prostate cancer • tissue microarray

Differentiating between indolent and aggressive prostate cancer (PC) is critical to optimal disease management [1,2]. The advent of prostate specific antigen (PSA) testing, while invaluable, has increased the potential for over-diagnosis and over-treatment [3,4]. Since all therapies carry some risks that may encompass life altering morbidity [5], good prognostic biomarkers are needed to identify those who could benefit from aggressive management [6]. Ideally, a useful biomarker adds prognostic information in a cost-effective manner. Ki67 is a well-studied protein exclusively expressed in proliferating cells throughout all cell cycle phases, except for G0 (the resting cycle) [7]. This allows for quick, easy and cost-effective determination of the 'growth-fraction' of cells. This property of Ki67 has generated substantial interest in using this antigen as a prognostic biomarker in cancer progression and metastasis [8].

Multiple studies [9-15] and a recent systematic review and meta-analysis [8] have provided compelling evidence for the prognostic value of Ki67 in determining the PC progression and disease-free survival risk. Although similar results were reported for breast cancer [16,17], the utility of Ki67 in breast cancer has been questioned due to its marginal reproducibility and lack of analytic standardization across laboratories $[18,19]$. It is noteworthy that most Ki67 studies in PC were conducted in single centers or in single pathology laboratories. Whether the inconsistencies reported in the reproducibility and validity of Ki67 expression scoring in breast cancer [18,19], also plague Ki67 estimation in PC is unknown.

We determined Ki67 expression using tissue microarrays (TMAs) of radical prostatectomy (RP) specimens from two centers. Our objectives were to determine if Ki67 expression predicted biochemical recurrence (BCR; primary outcome) and other markers of aggressive PC (e.g., castration resistant PC [CRPC], metastasis and PC-specific

Future : Medicine 
death; secondary outcomes). We hypothesized that while Ki67 would predict outcome in each center, the lack of standardization in Ki67 staining and scoring across centers would result in only modest prognostic value when the data were pooled.

\section{Materials \& methods Study population}

Approval was obtained for the construction of TMAs, data abstraction and analyses from the Internal Review Boards for Durham and West Los Angeles Veterans Affairs. RP specimens from 479 patients who underwent RP from the West Los Angeles Veterans Affairs Medical Center (WLAVAMC) and Durham, North Carolina Veterans Affairs Medical Center (DVAMC) between 1991 and 2004 and followed-up until November 2013 were used to construct TMAs. Exclusion criteria included preoperative hormonal ablation or radiation therapy. Eight patients missing follow-up and seven missing pathological data were excluded resulting in 464 subjects (WLAVAMC, $\mathrm{n}=293$; DVAMC, $\mathrm{n}=171$ ).

\section{Tissue acquisition/analysis Durham, North Carolina Veterans Affairs Medical Center}

From formalin fixed and paraffin embedded RP specimens, four core needle biopsies of cancer were used to construct TMAs. TMAs were sectioned into five micron sections, followed by immunohistochemical staining for Ki67 expression. A positive control tissue slide was included (human tonsil). The sections were dried for $30 \mathrm{~min}$ at $60^{\circ} \mathrm{C}$. Slides were deparaffinized in xylene, followed by a series of graded ethanol solutions, and rehydration in $\mathrm{dH} 20$ for $30 \mathrm{~s}$. Endogenous peroxidase activity was blocked with 3\% hydrogen peroxide for $10 \mathrm{~min}$. Slides were heated at $80^{\circ} \mathrm{C}$ in Dako citrate ( $\mathrm{pH}$ 6.1) antigen-retrieval buffer for $20 \mathrm{~min}$, cooled to room temperature, rinsed with water and placed in Tris-Buffered Saline and Tween 20 (TBST). Background Buster (Innovex Biosciences, CA, USA) was applied and incubated for $30 \mathrm{~min}$ at room temperature. The excess was drained without a wash. A 1:200 diluted rabbit monoclonal anti-Ki67 (Cat\# RM-9106-S1, Thermo Fisher Scientific, MA, USA) or rabbit $\mathrm{IgG}$, as a negative control antibody, was applied, incubated for $45 \mathrm{~min}$ at room temperature, then washed twice in TBST for $1 \mathrm{~min}$, and the excess buffer drained. A 1:200 diluted biotinylated secondary antibody (goat, antirabbit, Vector, CA, USA) was applied and incubated for $30 \mathrm{~min}$ followed by two $1 \mathrm{~min}$ washes with TBST. Vectastain Elite $\mathrm{ABC}$ was applied and incubated for $30 \mathrm{~min}$ followed again by two $1 \mathrm{~min}$ washes with TBST. The DAB chromogen (Innovex Biosciences, CA, USA) was applied and incubated for $3 \mathrm{~min}$, followed by three changes of $\mathrm{dH} 2 \mathrm{O}$, then rinsed with tap water for $2 \mathrm{~min}$. The sample was counter stained in hematoxylin for $30 \mathrm{~s}$ and rinsed with tap water. Slides were dipped four times in ammonium water and rinsed for $2 \mathrm{~min}$ to counterstain the nuclei in blue. Slides were dehydrated, cleared and coverslips were added.

\section{West Los Angeles Veterans Affairs Medical Center}

TMAs were constructed using a Manual Tissue Arrayer (Beecher Instruments, Inc., WI, USA). Coring needles having diameter $0.6 \mathrm{~mm}$ were used to abstract representative areas from formalin fixed paraffin embedded RP blocks. Tissue was sampled from the primary pathological Gleason grade, the secondary Gleason grade and benign tissue. Three cores of each histology type (primary Gleason grade, secondary Gleason grade and benign tissue) were taken from the blocks and placed into the TMA block. Four micron sections were cut from the TMA blocks and stained for Ki67, as previously described [20]. The slides were scanned using an Aperio slide scanner (Aperio, CA, USA).

\section{TMA reading}

TMAs were manually read by a board-certified pathologist at each respective center, blinded to clinical data and outcome. The average percent of positively stained cancer cells across the four core biopsies was calculated. At each site, 300 cancer cells were reviewed and the number of Ki67 positive cells were divided by 300 to compute the percent of positive cells. If the tissue was too scant for analysis, or no tumor was present, the core was considered nonevaluable and that core was excluded. The remaining cores were averaged.

\section{$B C R$ definition}

At both sites, BCR was defined as a single PSA value of $>0.2 \mathrm{ng} / \mathrm{ml}$, two values of $0.2 \mathrm{ng} / \mathrm{ml}$ or need for secondary treatment for elevated postoperative PSA. 


\section{Statistical analysis}

Since the distributions of percent Ki67 positive cells varied substantially between centers (Supplementary Figure 1), we divided subjects into tertiles of percent Ki67 positive staining within each center.

The patient demographic and clinical variables were compared using t-tests for continuous normally distributed variables, rank-sum for continuous non-normally distributed variables, and $\chi^{2}$ for categorical variables. Crude and multivariable Cox proportional hazard models were used to test the association between Ki67 tertile and BCR risk. Proportional hazard assumptions were examined and met for the center-specific and combined regression models using Schoenfeld residuals. Analyses were center-stratified as well as pooled. Multivariable analyses were adjusted for age (continuous), prognostic grade groups (GG I-V) [21], race (black/nonblack), surgery year (continuous), positive margins (yes/no), extracapsular extension (yes/no), seminal vesicle invasion (yes/no) and surgical center. An interaction between race and Ki67 was tested by including main effect terms and the cross-product, and evaluated using the Wald test. Kaplan-Meier curves were used to show the BCR-free survival between Ki67 tertiles by center. Statistical significance of these differences was assessed using the log-rank test.

On secondary analysis, we tested the prognostic value of Ki67 for other PC outcomes. We first examined the association between Ki67 and prognostic GG. We calculated median, 25th percentile, and 75th percentile of Ki67 positive staining values within each GG (I-V). To examine differences between centers, we calculated these values in both stratified by center and combined. Kruskal-Wallis test was used to test for an association between Ki67 and GG. We also used Cox regression models to test the association between Ki67 tertile and long-term PC outcomes: CRPC, metastases, and PC-specific death. CRPC was defined as having a PSA increase of $2 \mathrm{ng} / \mathrm{ml}$ and $25 \%$ greater than the nadir after hormone treatment despite continuous therapy with a luteinizing hormone-releasing hormone agonist, antagonist or after orchiectomy. Metastases were diagnosed by bone scan and other imaging. PC-specific death was defined as having metastatic progressive CRPC at death with no obvious other cause. Because event rates were low, centers were combined and all models were unadjusted.

To address potential concerns of tumor tissue under-sampling that could have led to misclassification of Ki67 status, we conducted sensitivity analyses where we excluded patients with negative Ki67 staining across all cores and repeated the above analyses.

\section{Results}

\section{Patient characteristics}

There were 215 black and 249 nonblack men in the study. There were no racial differences between surgical centers (Table 1) $(\mathrm{p}=0.27)$. DVAMC men were more likely to be treated more recently $(\mathrm{p}<0.001)$, have GG III or greater $(\mathrm{p}<0.001)$, positive margins $(\mathrm{p}<0.001)$, extracapsular extension $(\mathrm{p}<0.001)$ and higher Ki67 scores $(\mathrm{p}<0.001)$. There were no differences between centers in age, preoperative PSA, seminal vesicle invasion, positive nodes or mean follow-up (all p-values $>0.18$ ).

We did not find associations between Ki67 tertiles and age, PSA and GG (Table 2). However, a lower percentage of men were classified in pathological stage T0-T2 (67\%) in tertile 3 compared with tertiles 1 and $2(\mathrm{p}=0.010)$.

\section{Center specific risk of $B C R$}

Median PSA follow-up times were 115 months (Q1-Q3: 56-162 months) and 117 months (Q1-Q3: 79144 months) for WLAVAMC and DVAMC, respectively. During follow-up, 200 (43\%) patients had BCR: 122 patients from WLAVAMC and 78 from DVAMC. In WLAVAMC, BCR was not significantly related to Ki67 tertile ( $\log$-rank, $p=0.205)$ (Figure 1A). However, there was an increasing risk with increasing tertile which approached but did not reach statistical significance for the highest versus lowest tertile in crude (hazard ratio [HR]: 1.48; 95\% CI: 0.96-2.28; $\mathrm{p}=0.08$ ) and adjusted analyses (HR: 1.54; 95\% CI: 0.98-2.44; $\mathrm{p}=0.064$ ) (Table 3).

Within the DVAMC cohort, Ki67 tertile was significantly related to BCR, but the second tertile actually had the best outcome (log-rank; $\mathrm{p}=0.006$ ) (Figure $1 \mathrm{~B})$. When examined in two-way comparisons, the second tertile was associated with a significantly lower BCR risk in crude analysis (HR: 0.55; $\mathrm{p}=0.0496$ ), which was attenuated in multivariable analysis (HR: 0.64; $\mathrm{p}=0.17$ ). The highest tertile was unrelated to BCR in both crude and adjusted analyses (all p-value $>0.2$ ).

\section{$\mathrm{BCR}$ risk in pooled data}

When the data from both centers were combined, Ki67 tertile was significantly associated with BCR with men in the third tertile having the worst outcomes (log-rank, $p=0.009$, Figure 1C). Indeed, men in the third Ki67 tertile 


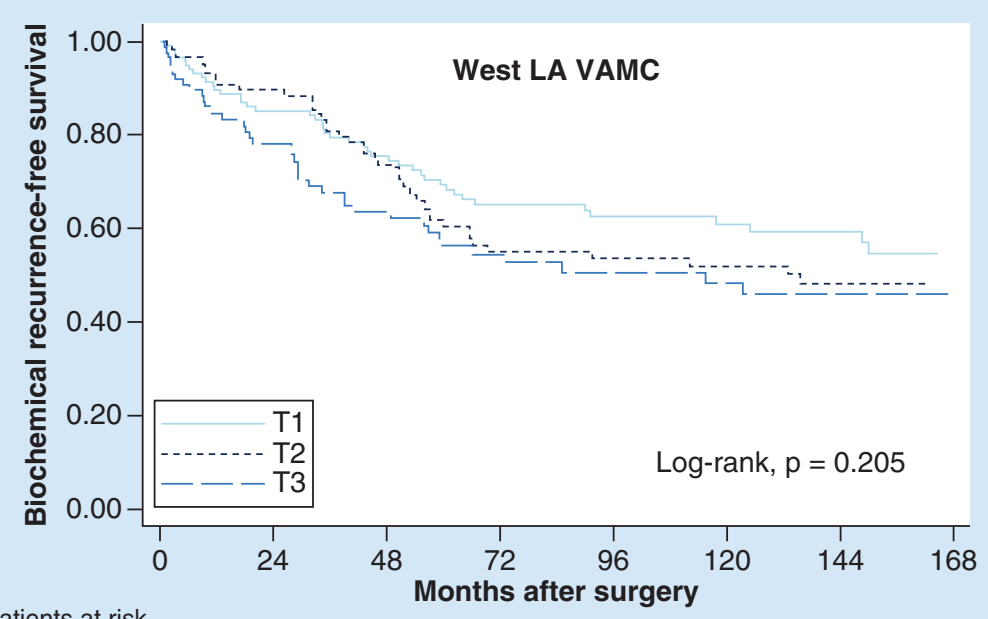

\begin{tabular}{|c|c|c|c|c|c|c|}
\hline & & & 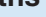 & 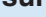 & & \\
\hline \# Patients at risk & & & & & & \\
\hline T1 119 & 93 & 76 & 57 & 46 & 37 & 27 \\
\hline T2 87 & 73 & 58 & 38 & 37 & 31 & 22 \\
\hline T3 87 & 60 & 44 & 30 & 24 & 21 & 17 \\
\hline
\end{tabular}

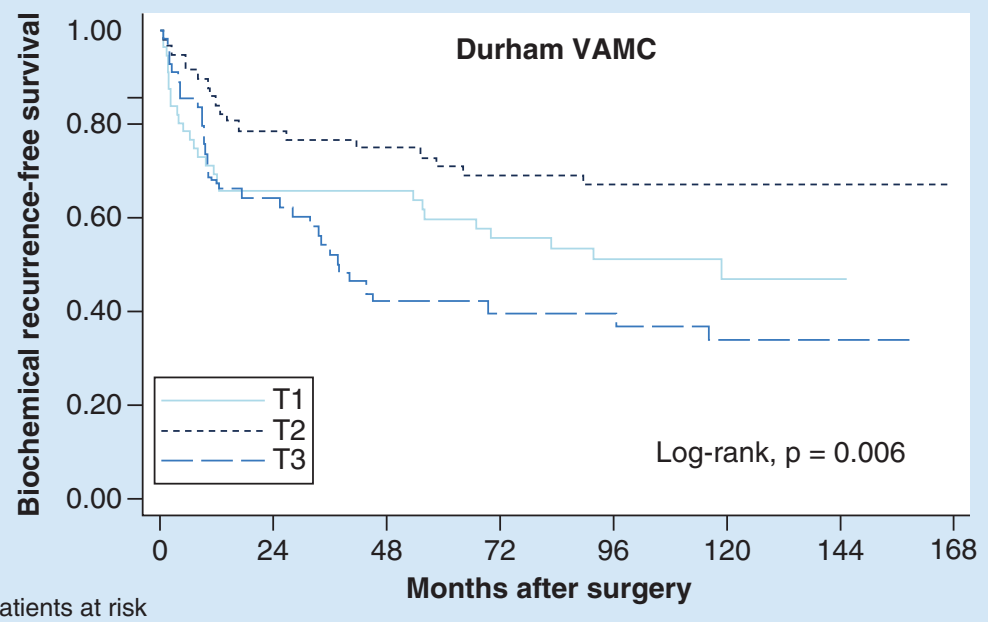

\# Patients at risk

$\begin{array}{lllllllll}\text { T1 } & 56 & 36 & 34 & 28 & 19 & 10 & 4 & 2 \\ \text { T2 } & 58 & 43 & 40 & 36 & 33 & 21 & 13 & 7 \\ \text { T3 } & 57 & 32 & 19 & 16 & 15 & 10 & 7 & 4\end{array}$

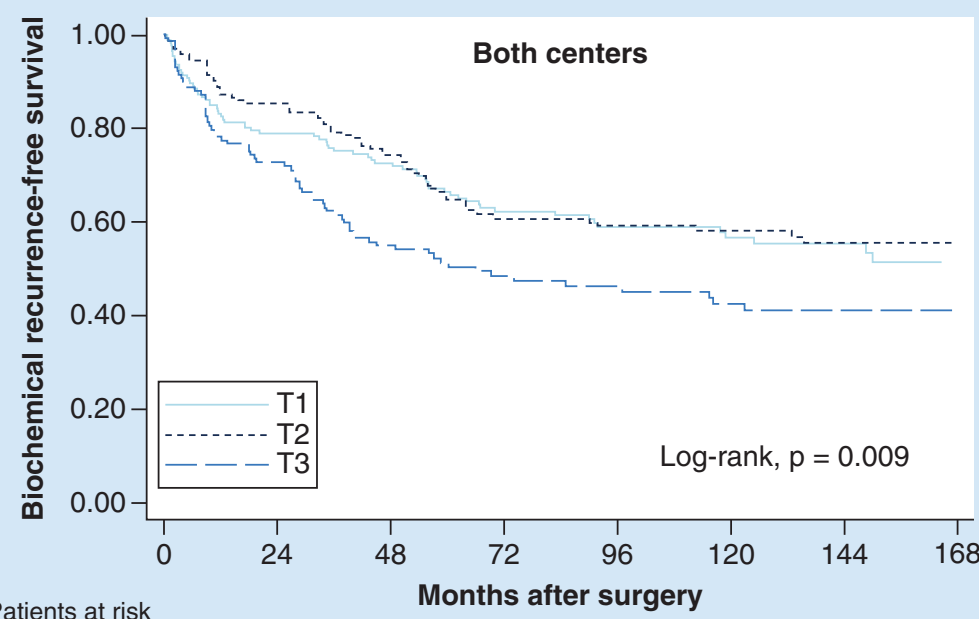

\# Patients at risk

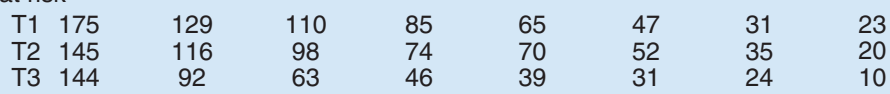


Table 1. Demographic, clinical and pathological characteristics of patients by surgical center.

\begin{tabular}{|c|c|c|c|}
\hline Patient characteristics & WLAVAMC $(n=293)$ & DVAMC $(n=171)$ & $p$-value \\
\hline Age, mean $\pm S D$ & $62.8 \pm 6.3$ & $62.3 \pm 6.2$ & $0.39^{\dagger}$ \\
\hline \multicolumn{3}{|l|}{ Race, $\mathrm{n}(\%)$ : } & $0.27^{\ddagger}$ \\
\hline - Nonblack & $163(56)$ & $86(50)$ & \\
\hline Year of surgery, M (Q1-Q3) & 1997 (1994-1999) & $2000(1997-2002)$ & $<0.001^{\S}$ \\
\hline PSA (ng/ml), M (Q1-Q3) & $8.4(5.5-12.6)$ & $7.7(5.1-12.0)$ & $0.31^{\S}$ \\
\hline$-2-3$ & $89(30)$ & $89(52)$ & \\
\hline$-4-5$ & $45(15)$ & $46(27)$ & \\
\hline Positive margins, $\mathrm{n}(\%)$ & $136(46)$ & $108(63)$ & $<0.001^{\ddagger}$ \\
\hline Seminal vesicle invasion, $\mathrm{n}(\%)$ & $32(11)$ & $26(15)$ & $0.18^{\ddagger}$ \\
\hline Extracapsular extension, n (\%) & $30(10)$ & $47(27)$ & $<0.001^{\ddagger}$ \\
\hline - Tertile 1, minimum-maximum & $0-0$ & $0-1.66$ & \\
\hline - Tertile 2, minimum-maximum & $0.03-0.50$ & $1.67-4.58$ & \\
\hline - Tertile 3, minimum-maximum & $0.53-10.29$ & $4.67-18.67$ & \\
\hline Follow-up (months), M (Q1-Q3) & $115(56-162)$ & $117(79-144)$ & 0.83 \\
\hline \multicolumn{4}{|c|}{$\begin{array}{l}\text { †p-values calculated using t-test. } \\
\text { ¥p-values calculated using } \chi^{2} \text { test. } \\
\text { }_{\mathrm{p} \text {-values calculated using rank sum test. }} \\
\text { DVAMC: Durham, North Carolina Veterans Affairs Medical Center; M: Median; PSA: Prostate specific antigen; Q1: 25th percentile; Q3: 75th percentile; SD: Standard deviation; WLAVAMC: } \\
\text { West Los Angeles Veterans Affairs Medical Center. }\end{array}$} \\
\hline
\end{tabular}

\begin{tabular}{|c|c|c|c|c|}
\hline Patient characteristics & Ki67 tertile $1(n=175)$ & Ki67 tertile $2(n=145)$ & Ki67 tertile $3(n=144)$ & $p$-value ${ }^{\dagger}$ \\
\hline Age, median (IQR) & $64(59-68)$ & $63(58-66)$ & $64(59-68)$ & 0.30 \\
\hline \multicolumn{4}{|c|}{ Post-op grade group, n (\%): } & 0.49 \\
\hline-1 & $83(47)$ & $56(39)$ & $56(39)$ & \\
\hline$-2-3$ & $81(46)$ & $79(55)$ & $77(53)$ & \\
\hline \multicolumn{4}{|c|}{ Pathological stage, n (\%): } & 0.010 \\
\hline$-\mathrm{TO}-\mathrm{T} 2$ & $141(81)$ & $117(81)$ & $96(67)$ & \\
\hline$-\mathrm{T} 3$ & $25(14)$ & $25(17)$ & $41(28)$ & \\
\hline$-\mathrm{T} 4$ & $9(5)$ & $3(2)$ & $7(5)$ & \\
\hline
\end{tabular}

${ }^{\dagger} p$-values calculated using Kruskal-Wallis for age and PSA and $\chi^{2}$ test for grade group and pathological stage. IQR: Interquartile range; PSA: Prostate specific antigen.

had a statistically significant increased BCR risk versus men in the first tertile on crude analysis (HR: 1.50; 95\% CI: 1.08-2.09; $\mathrm{p}=0.015$ ) (Table 4). After multivariable adjustment, the HR was attenuated and no longer significant (HR: 1.33; 95\% CI: 0.94-1.89; $\mathrm{p}=0.11$ ) (Table 4). The interaction term for race and Ki67 was not statistically significant ( $\mathrm{p}$-interaction $=0.56$ ); hence, the relation between Ki67 and BCR was not further stratified by race.

\section{Long-term secondary outcomes}

During a median overall follow-up of 117 and 115 months, in DVAMC and WLAVAMC, respectively, 26 men developed CRPC, 29 developed metastases, and 18 PC deaths occurred. When stratified by site, Ki67 was significantly associated with GG in DVAMC ( $\mathrm{p}=0.043)$, but not WLAVAMC $(\mathrm{p}=0.62)$ (Supplementary Table 1). In pooled analyses, Ki67 was significantly associated with GG $(\mathrm{p}<0.001)$. The associations between Ki67 
Table 3. Hazard ratios for risk of biochemical recurrence by tertile of percent of cancer cells staining positive for Ki67

\section{stratified by surgical center.}

\begin{tabular}{|c|c|c|c|c|c|c|}
\hline \multirow[t]{2}{*}{ Tertile of Ki67 } & \multicolumn{3}{|c|}{ WLAVAMC $(n=293)$} & \multicolumn{3}{|c|}{ DVAMC $(n=171)$} \\
\hline & $\mathrm{BCR} / \mathrm{N}$ & $\mathrm{HR}(95 \% \mathrm{Cl})$ & p-value & $\mathrm{BCR} / \mathrm{N}$ & HR $(95 \% \mathrm{Cl})$ & p-value \\
\hline \multicolumn{7}{|l|}{ Crude: } \\
\hline$-\mathrm{T} 1$ & $43 / 119$ & Reference & & $27 / 56$ & Reference & \\
\hline$-\mathrm{T} 2$ & $40 / 87$ & $1.23(0.80-1.89)$ & 0.35 & $18 / 58$ & $0.55(0.30-0.999)$ & 0.0496 \\
\hline$-\mathrm{T} 3$ & $39 / 87$ & $1.48(0.96-2.28)$ & 0.08 & $33 / 57$ & $1.38(0.83-2.31)$ & 0.21 \\
\hline \multicolumn{7}{|l|}{ 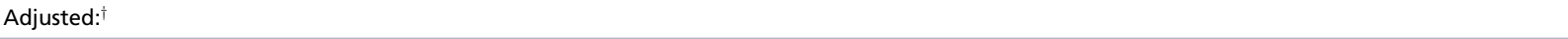 } \\
\hline$-\mathrm{T} 1$ & $43 / 119$ & Reference & & $27 / 56$ & Reference & \\
\hline$-\mathrm{T} 2$ & $40 / 87$ & $1.01(0.64-1.60)$ & 0.95 & $18 / 58$ & $0.64(0.34-1.21)$ & 0.17 \\
\hline$-\mathrm{T} 3$ & $39 / 87$ & $1.54(0.98-2.44)$ & 0.064 & $33 / 57$ & $1.10(0.63-1.94)$ & 0.74 \\
\hline
\end{tabular}

\begin{tabular}{|c|c|c|c|}
\hline Tertile of Ki67 & $\mathrm{BCR} / \mathrm{N}$ & HR $(95 \% \mathrm{Cl})$ & p-value \\
\hline \multicolumn{4}{|l|}{ Crude: } \\
\hline$-\mathrm{T} 1$ & $70 / 175$ & Reference & \\
\hline$-\mathrm{T} 2$ & $58 / 145$ & $0.92(0.65-1.31)$ & 0.66 \\
\hline$-\mathrm{T3}$ & $72 / 144$ & $1.50(1.08-2.09)$ & 0.015 \\
\hline \multicolumn{4}{|l|}{ Adjusted: $^{\dagger}$} \\
\hline$-\mathrm{T} 1$ & $70 / 175$ & Reference & \\
\hline$-\mathrm{T} 2$ & $58 / 145$ & $0.88(0.62-1.26)$ & 0.43 \\
\hline$-\mathrm{T} 3$ & $72 / 144$ & $1.33(0.94-1.89)$ & 0.11 \\
\hline
\end{tabular}

tertiles and all long-term outcomes were null on univariable analysis $(\mathrm{p} \geq 0.65)$ (Supplementary Table 2) (there were an insufficient number of events to perform multivariable analyses).

\section{Analyses excluding patients with no positive Ki67 staining}

There were 119 patients at WLAVAMC and 14 patients at DVAMC with no Ki67 staining; following their exclusion 331 remained. Results were similar to those observed for the entire sample (Supplementary Tables $3 \&$ 4).

\section{Comments}

Ki67 is a marker of cellular proliferation expressed in all but the G0 phase of the cell cycle. Since uncontrolled proliferation is a hallmark of cancer [22], Ki67 expression has generated substantial interest for its potential as a cancer biomarker $[8,23]$. Although it shows promise in determining the aggressiveness of breast and prostate cancers [9-17], its quantification is compromised by poor reproducibility $[18,19,24]$. This limitation raises questions with respect to its clinical utility and prompted our interest in evaluating its performance in predicting PC aggressiveness across two institutions where TMAs were evaluated by different pathology laboratories. Null to weak associations were observed between Ki67 and BCR in center-stratified and pooled analyses, while no associations were observed between Ki67 and long-term PC outcomes: CRPC, metastases and PC-specific death. Although these findings do not rule out an association between Ki67 expression and aggressive PC, they suggest a modest association of limited clinical utility. Importantly our results demonstrate the substantial variability in immunohistological staining and scoring between centers under real-world conditions. As such, they further substantiate concerns pertaining to the application of Ki67 to current clinical practice. 
The over-riding implications of our findings are that there is a lack of standardization in the technical and analytical methods used to quantify Ki67 expression. Manual and automated laboratory methods are available for the determination of Ki67 staining with each method currently associated with distinct advantages and inherent limitations. In this study, a board-certified pathologist manually counted cells for positive KI67 staining. Although feasible in most clinical centers, this approach is known to be subject to intra- and interobserver variability [25] which likely played a role in our discrepant Ki67 distributions. In addition, the method is limited by the number of cells that can realistically be counted in larger studies without over-extending resources. We included 300 cells per core in the evaluation of Ki67 status. However, manual counting of as many as 1000 cells has been suggested to optimally evaluate Ki67 status [18]. This may be too labor-intensive for most laboratories. Automated digital image analysis is an alternate approach that is becoming more accessible and is proposed for increasing the precision, reproducibility and rapidity of Ki67quantification [25]. The technology is promising, but remains limited by its poor ability to distinguish lymphocytes from tumor cells. On the other hand, with visual inspection, lymphocytes are easily differentiated from tumor cells, thus avoiding the potential for misclassified [26]. In prostate cancer studies, a comparison of digital image analysis with visual assessment has led to conflicting results with respect to the concordance of Ki67 staining and its relation to PC outcomes [25,27]. Similarly, in breast cancer, superiority of one method over the other has not emerged, prompting an international multicenter collaboration to focus on the standardization of Ki67 expression scoring [24,28]. Clearly, standardization of a readily available Ki67 staining quantification method is essential prior to its widespread use as a cancer biomarker.

Another issue critical to the clinical utility of Ki67 as a cancer biomarker is the establishment of threshold cutpoints that risk-stratify patients for PC outcomes. Although Ki67 expression is a continuous measure $[18,23,29]$ that is often studied as such in relation to cancer-related outcomes [25], continuous markers do not readily translate into clinical decisions that align with treatment strategies. In the absence of consensus pertaining to universal cut-points, we classified subjects by Ki67 tertiles. The differences in Ki67 score distributions between centers resulted in vastly different tertile cut-points. A weak association between Ki67 and BCR was observed for WLAVAMC where the upper tertile cut-point was $0.53 \%$, while the results were null for DVAMC where the upper tertile cut-point was 4.67\%. In PC studies with dichotomized Ki67 scores, cut-points between 1 and 26\% have been used to examine the relation between Ki67 and BCR [11,14,15] and between 1 and $11.3 \%$ for disease-specific survival [8,9]. Studies that have used multiple cut-points indicate that cut-points of $10 \%$ or lower may be most effective [12-14]. Although the percent cut-points for the highest tertiles in this study are in line with those reported in recent studies, we observed predominantly null to borderline statistically significant associations for the relation between Ki67 and BCR.

Beyond the issues discussed above, if Ki67 is to be of clinical utility, its measurement must demonstrate robust prognostic value. Notably, two separate recent multicenter studies examined Ki67 and outcomes after RP (similar to our study) and found Ki67 predicted recurrence (unlike our study) [13,15]. However, there are notable differences between these studies and ours. First, the studies performed Ki67 staining and scoring in single laboratories, thereby eliminating differences between laboratories. Although studying the biomarker potential of Ki67 under optimal conditions is appropriate, this approach is not indicative of 'real-world' practice as exemplified in our study wherein tissue preparation, staining and reading were done separately at each center. Second, these two studies had larger sample sizes $(n=1004$ and $n=3123)$ than ours $(n=464)$. If a biomarker requires $>1000$ men to demonstrate clinical significance, the clinical utility is likely low. As such, though our study was smaller, it was sufficiently powered to detect clinically meaningful differences. Indeed, in the larger of the two studies ( $\mathrm{n}=3123)$, a dichotomized Ki67 score $\geq 20 \%$ increased recurrence risk by a mere $19 \%$ in multivariable analysis $(\mathrm{p}=0.019)$ [15]. 3-year BCR-free rates were 85 versus $82.1 \%$ - again a statistically significant difference, but one with minimal clinical utility. In the second study $(\mathrm{n}=1004)$, the effect of Ki67 was larger, but nonetheless modest [13]. A Ki67 score $>5 \%$ increased the relative risk of recurrence by $47 \%(\mathrm{p}=0.007)$, again a threshold that is unlikely to have significant effects on overall risk stratification. Third, the cut-points used in the two prior multicenter studies differed (5 and 20\%) further demonstrating the lack of consensus with respect to how to use Ki67 clinically. In summary, even when analyzed in a single laboratory, the clinical utility of Ki67 appears to be limited at best.

A major strength of our study is that we conducted a multicenter analysis with noncentralized Ki67 immunostaining and scoring, in contrast to larger studies where single laboratories with specially trained genitourinary pathologists were employed, often with sophisticated imaging technologies [13,15]. Our methods resemble contemporary 'real-world' scenarios. In addition, all patients in this study underwent RP, and the results are most relevant 
to this group. Additional strengths of the study are the long follow-up period and the multiethnic mix of patients coming from two different centers.

Of note, there are limitations in our study. First, there was no centralized determination of the prognostic GG of RP specimens or standardization of Ki67 tissue acquisition, preparation or analysis. However, this allowed us to illustrate the impact of unstandardized methods on the determination of Ki67 expression. Second, our sample size was limited by the number of TMAs available from the centers and may have limited our power to detect true associations between Ki67 and PC outcomes.

Clearly there is a need for robust clinically feasible biomarkers that identify patients with high-risk disease and those with high risk of recurrence. It is with this aim that prognostic biomarkers continue to be investigated. For example, while there has been interest in the prognostic ability of premalignant prostatic lesions such as high-grade prostatic intraepithelial neoplasia (HGPIN), a recent review, concluded that the risk of cancer after a diagnosis of HGPIN is about $20-30 \%$. This is similar to the risk of cancer on repeat needle biopsy among men with benign diagnosis on initial biopsy [30]. Nonetheless, the data to date are limited, warranting ongoing research into the potential role of HGPIN in the identification of patients with high risk disease.

Given the limited clinical utility of Ki67, it is incumbent on the field to identify valuable markers. Current efforts are focused on multiplex markers, often based upon genomic testing. Other markers, however, should be explored either alone or in combination such as $\mathrm{p} 53$, centrosomal protein 57, phosphatase and tensin homolog, and $\mathrm{p} 27$.

\section{Conclusion}

The PC field continues to be rife with decisions on treatment options and the need for further risk stratification remains paramount. There is an urgent need for biomarker-driven clinical decisions to better define surveillance protocols and specifically to determine the prognostic outcomes of those having received curative surgery [8]. We performed a multi-institutional analysis of the Ki67 expression assay as a marker for aggressive PC using BCR as our end point. In this study, Ki67 was not associated with a higher risk for developing BCR, in part owing to the great Ki67 expression variation between centers. To truly examine the clinical utility of Ki67 in assessing PC aggressiveness, standardization of staining, reading, and analysis, readily available in 'real world' clinical settings is required.

\section{Summary points}

- There is an urgent need to identify prognostic biomarkers that differentiate between indolent and highly aggressive prostate cancers.

- Ki67 has generated substantial interest as a prognostic biomarker in cancer progression.

- Poor reproducibility and lack of standardization raise questions regarding its clinical utility.

- Two pathology laboratories were used for the construction of tissue microarrays, immunohistochemical staining and Ki67 expression scoring.

- Prostate cancer outcomes were assessed after a median of about 10 years of follow-up.

- Null to weak associations were observed between Ki67 and biochemical recurrence.

- Ki67 was not associated with castration-resistant prostate cancer, metastases and prostate cancer-specific mortality.

- We observed substantial variability in immunohistological staining and scoring between centers.

- This 'real-world' study substantiates current concerns pertaining to the application of Ki67 to clinical practice.

Supplementary data

To view the supplementary data that accompany this paper please visit the journal website at: www.futuremedicine.com/doi/full/10.2217/bmm-2017-0322

Financial \& competing interests disclosure

Support for this study was provided by the NIH/NCI under Award Number P50CA09231 and NIH K24 CA160653. The authors have no other relevant affiliations or financial involvement with any organization or entity with a financial interest in or financial conflict with the subject matter or materials discussed in the manuscript apart from those disclosed.

No writing assistance was utilized in the production of this manuscript. 


\section{Ethical conduct of research}

The authors state that they have obtained appropriate institutional review board approval or have followed the principles outlined in the Declaration of Helsinki for all human or animal experimental investigations. In addition, for investigations involving human subjects, informed consent has been obtained from the participants involved.

\section{References}

Papers of special note have been highlighted as: $\bullet \bullet$ of considerable interest

1. Canfield SE, Kibel AS, Kemeter MJ, Febbo PG, Lawrence HJ, Moul JW. A guide for clinicians in the evaluation of emerging molecular diagnostics for newly diagnosed prostate cancer. Rev. Urol. 16(4), 172-180 (2014).

2. Litwin MS, Tan H. The diagnosis and treatment of prostate cancer: a review. J. Am. Med. Assoc. 317(24), 2532-2542 (2017).

-. Summary of recent advances in prostate cancer diagnosis and treatment.

3. Etzioni R, Penson DF, Legler JM et al. Overdiagnosis due to prostate-specific antigen screening: lessons from U.S. prostate cancer incidence trends. J. Natl Cancer Inst. 94(13), 981-990 (2002).

4. Gulati R, Inoue LY, Gore JL, Katcher J, Etzioni R. Individualized estimates of overdiagnosis in screen-detected prostate cancer. J. Natl Cancer Inst. 106(2), djt367 (2014).

5. Brajtbord JS, Punnen S, Cowan JE, Welty CJ, Carroll PR. Age and baseline quality of life at radical prostatectomy - who has the most to lose? J. Urol. 192(2), 396-401 (2014).

6. Gillessen S, Attard G, Beer TM et al. Management of patients with advanced prostate cancer: the report of the Advanced Prostate Cancer Consensus Conference APCCC 2017. Eur. Urol. 73(2), 187-211 (2018).

7. Scholzen T, Gerdes J. The Ki-67 protein: from the known and the unknown. J. Cell. Physiol. 182(3), 311-322 (2000).

8. Berlin A, Castro-Mesta JF, Rodriguez-Romo L et al. Prognostic role of Ki-67 score in localized prostate cancer: a systematic review and meta-analysis. Urol. Oncol. 35(8), 499-506 (2017)

-• Includes a detailed overview of the variations in Ki67 staining cut-points for classifying risk.

9. Berney DM, Gopalan A, Kudahetti S et al. Ki-67 and outcome in clinically localised prostate cancer: analysis of conservatively treated prostate cancer patients from the Trans-Atlantic Prostate Group study. Br. J. Cancer 100(6), 888-893 (2009).

-• Large study compared various Ki67 cut-points within same sample.

10. Keshgegian AA, Johnston E, Cnaan A. Bcl-2 oncoprotein positivity and high MIB-1 (Ki-67) proliferative rate are independent predictive markers for recurrence in prostate carcinoma. Am. J. Clin. Pathol. 110(4), 443-449 (1998).

11. Green WJF, Ball G, Hulman G et al. KI67 and DLX2 predict increased risk of metastasis formation in prostate cancer - a targeted molecular approach. Br. J. Cancer 115(2), 236-242 (2016).

12. Pascale M, Aversa C, Barbazza R et al. The proliferation marker Ki67, but not neuroendocrine expression, is an independent factor in the prediction of prognosis of primary prostate cancer patients. Radiol. Oncol. 50(3), 313-320 (2016).

13. Tretiakova MS, Wei W, Boyer HD et al. Prognostic value of Ki67 in localized prostate carcinoma: a multi-institutional study of $>1000$ prostatectomies. Prostate Cancer Prostatic. Dis. 19(3), 264-270 (2016).

-. Thorough presentation of methods and discussion of potential application of Ki67 as a biomarker in clinical practice.

14. Fisher G, Yang ZH, Kudahetti S et al. Prognostic value of Ki-67 for prostate cancer death in a conservatively managed cohort. Br.J. Cancer 108(2), 271-277 (2013).

15. Mathieu R, Shariat SF, Seitz C et al. Multi-institutional validation of the prognostic value of Ki-67 labeling index in patients treated with radical prostatectomy. World J. Urol. 33(8), 1165-1171 (2015).

16. Yerushalmi R, Woods R, Ravdin PM, Hayes MM, Gelmon KA. Ki67 in breast cancer: prognostic and predictive potential. Lancet Oncol. 11(2), 174-183 (2010).

17. De Azambuja E, Cardoso F, De Castro G Jr et al. Ki-67 as prognostic marker in early breast cancer: a meta-analysis of published studies involving 12,155 patients. Br. J. Cancer 96(10), 1504-1513 (2007).

18. Dowsett M, Nielsen TO, A'Hern R et al. Assessment of Ki67 in breast cancer: recommendations from the International Ki67 in Breast Cancer Working Group. J. Natl Cancer Inst. 103(22), 1656-1664 (2011).

19. Polley MY, Leung SC, McShane LM et al. An international Ki67 reproducibility study. J. Natl Cancer Inst. 105(24), 1897-1906 (2013).

20. Kobayashi N, Barnard RJ, Henning SM et al. Effect of altering dietary $\omega-6 / \omega-3$ fatty acid ratios on prostate cancer membrane composition, cyclooxygenase-2, and prostaglandin E2. Clin. Cancer Res. 12(15), 4662-4670 (2006).

21. Epstein JI, Zelefsky MJ, Sjoberg DD et al. A contemporary prostate cancer grading system: a validated alternative to the Gleason score. Eur. Urol. 69(3), 428-435 (2016).

22. Hanahan D, Weinberg RA. Hallmarks of cancer: the next generation. Cell 144(5), 646-674 (2011).

23. Denkert C, Budczies J, Von Minckwitz G, Wienert S, Loibl S, Klauschen F. Strategies for developing Ki67 as a useful biomarker in breast cancer. Breast 24, S67-S72 (2015). 
•• Review of lessons learned from decades of studies examining the prognostic value of Ki67 in breast cancer.

24. Polley M-YC, Leung SCY, Gao D et al. An international study to increase concordance in Ki67 scoring. Mod. Pathol. 28(6), 778-786 (2015).

25. Desmeules P, Hovington H, Nguilé-Makao M et al. Comparison of digital image analysis and visual scoring of KI-67 in prostate cancer prognosis after prostatectomy. Diagn. Pathol. 10(1), 67 (2015).

26. Zhong F, Bi R, Yu B, Yang F, Yang W, Shui R. A comparison of visual assessment and automated digital image analysis of Ki67 labeling index in breast cancer. PLoS ONE 11(2), e0150505 (2016).

27. Verhoven B, Yan Y, Ritter M et al. Ki-67 is an independent predictor of metastasis and cause-specific mortality for prostate cancer patients treated on Radiation Therapy Oncology Group (RTOG) 94-08. Int. J. Radiat. Oncol. Biol. Phys. 86(2), 317-323 (2013).

28. Leung SCY, Nielsen TO, Zabaglo L et al. Analytical validation of a standardized scoring protocol for Ki67: Phase 3 of an international multicenter collaboration. NPJ Breast Cancer 18(2), 16014 (2016).

29. Cserni G, Vörös A, Liepniece-Karele I et al. Distribution pattern of the Ki67 labelling index in breast cancer and its implications for choosing cut-off values. Breast 23(3), 259-263 (2014).

30. Tosoian JJ, Alam R, Ball MW, Carter HB, Epstein JI. Managing high-grade prostatic intraepithelial neoplasia (HGPIN) and atypical glands on prostate biopsy. Nat. Rev. Urol. 15, 55 (2017). 